\title{
OGLE-2018-BLG-0567Lb and OGLE-2018-BLG-0962Lb: Two Microlensing Planets through the Planetary-caustic Channel
}

Youn Kil Jung $^{1,2,15}$ (D), Cheongho $\operatorname{Han}^{3,15}$ (D), Andrzej Udalski ${ }^{4,16}$ (D), Andrew Gould ${ }^{1,5,6,15}$, Jennifer C. Yee ${ }^{7,15}$ (D) and

Michael D. Albrow ${ }^{8}$ (D), Sun-Ju Chung ${ }^{1,2}$ (D) , Kyu-Ha Hwang ${ }^{1}$ (D), Yoon-Hyun Ryu ${ }^{1}$ (D), In-Gu Shin ${ }^{1}$ (D), Yossi Shvartzvald ${ }^{9}$ (D), Wei Zhu ${ }^{10}$, Weicheng Zang ${ }^{11}$ (D), Sang-Mok Cha ${ }^{1,12}$, Dong-Jin Kim ${ }^{1}$, Hyoun-Woo Kim ${ }^{1}$ (D), Seung-Lee Kim ${ }^{1,2} \mathbb{D}^{(\mathbb{D}}$, Chung-Uk Lee ${ }^{1,2}$ (D) , Dong-Joo Lee ${ }^{1}$, Yongseok Lee ${ }^{1,12}$, Byeong-Gon Park ${ }^{1,2}$ (D), Richard W. Pogge ${ }^{5}$ (iD

(The KMTNet Collaboration),

and

Przemek Mróz ${ }^{4,13}$ (D), Michał K. Szymański ${ }^{4}$ (D) Jan Skowron $^{4}$ (D), Radek Poleski ${ }^{4,5}$, Igor Soszyński ${ }^{4}$ (D), Paweł Pietrukowicz ${ }^{4}$ (iD), Szymon Kozłowski ${ }^{4}$ (D), Krzystof Ulaczyk ${ }^{14}$ (D), Krzysztof A. Rybicki ${ }^{4}$, Patryk Iwanek ${ }^{4}$ (D), and Marcin Wrona ${ }^{4}$

(The OGLE Collaboration)

\author{
${ }^{1}$ Korea Astronomy and Space Science Institute, Daejon 34055, Republic of Korea \\ ${ }^{2}$ University of Science and Technology, Korea, 217 Gajeong-ro Yuseong-gu, Daejeon 34113, Korea \\ ${ }^{3}$ Department of Physics, Chungbuk National University, Cheongju 28644, Republic of Korea \\ ${ }^{4}$ Warsaw University Observatory, Al. Ujazdowskie 4, 00-478 Warszawa, Poland \\ ${ }^{5}$ Department of Astronomy, Ohio State University, 140 W. 18th Ave., Columbus, OH 43210, USA \\ ${ }^{6}$ Max-Planck-Institute for Astronomy, Königstuhl 17, D-69117 Heidelberg, Germany \\ ${ }^{7}$ Center for Astrophysics|Harvard \& Smithsonian, 60 Garden St., Cambridge, MA 02138, USA \\ ${ }^{8}$ University of Canterbury, Department of Physics and Astronomy, Private Bag 4800, Christchurch 8020, New Zealand \\ ${ }^{9}$ Department of Particle Physics and Astrophysics, Weizmann Institute of Science, Rehovot 76100, Israel \\ ${ }^{10}$ Canadian Institute for Theoretical Astrophysics, University of Toronto, 60 St. George St., Toronto, ON M5S 3H8, Canada \\ ${ }^{11}$ Department of Astronomy, Tsinghua University, Beijing 100084, People's Republic of China \\ ${ }^{12}$ School of Space Research, Kyung Hee University, Yongin 17104, Republic of Korea \\ ${ }^{13}$ Division of Physics, Mathematics, and Astronomy, California Institute of Technology, Pasadena, CA 91125, USA \\ ${ }^{14}$ Department of Physics, University of Warwick, Gibbet Hill Road, Coventry, CV4 7AL, UK \\ Received 2021 January 31; revised 2021 March 23; accepted 2021 April 14; published 2021 June 3
}

\begin{abstract}
We present the analyses of two microlensing events, OGLE-2018-BLG-0567 and OGLE-2018-BLG-0962. In both events, the short-lasting anomalies were densely and continuously covered by two high-cadence surveys. The lightcurve modeling indicates that the anomalies are generated by source crossings over the planetary caustics induced by planetary companions to the hosts. The estimated planet/host separation (scaled to the angular Einstein radius $\theta_{\mathrm{E}}$ ) and mass ratio are $\left(s, q \times 10^{3}\right)=(1.81 \pm 0.02,1.24 \pm 0.07)$ and $\left(s, q \times 10^{3}\right)=(1.25 \pm 0.03,2.38 \pm 0.08)$, respectively. From Bayesian analyses, we estimate the host and planet masses as $\left(M_{\mathrm{h}}, M_{\mathrm{p}}\right)=\left(0.25_{-0.13}^{+0.27} M_{\odot}, 0.32_{-0.17}^{+0.34} M_{\mathrm{J}}\right)$ and $\left(M_{\mathrm{h}}, M_{\mathrm{p}}\right)=\left(0.54_{-0.28}^{+0.33} M_{\odot}, 1.34_{-0.70}^{+0.82} M_{\mathrm{J}}\right)$, respectively. These planetary systems are located at a distance of $7.06_{-1.15}^{+0.93} \mathrm{kpc}$ for OGLE-2018-BLG-0567 and 6.50 $-1.06 \mathrm{kpc}$ for OGLE-2018-BLG-0962, suggesting that they are likely to be near the Galactic bulge. The two events prove the capability of current high-cadence surveys for finding planets through the planetary-caustic channel. We find that most published planetary-caustic planets are found in Hollywood events in which the source size strongly contributes to the anomaly cross-section relative to the size of the caustic.
\end{abstract}

Unified Astronomy Thesaurus concepts: Gravitational microlensing (672); Gravitational microlensing exoplanet detection (2147)

Supporting material: data behind figures

\section{Introduction}

The signature of a microlensing planet is almost always a short-lasting anomaly in the smooth and symmetric lensing light curve produced by the host of the planet. In principle, the signature can appear at any position of the lensing light curve (Gaudi 2012). In reality, however, the signatures of planets detected in the earlier phase of lensing experiments appeared mainly near the peak of lensing light curves.

The bias toward central anomalies is mostly attributed to the limitation of early lensing surveys. With a roughly 1-day

\footnotetext{
15 The KMTNet Collaboration

16 The OGLE Collaboration.
}

cadence of the first-generation survey experiments, e.g., the Massive Compact Halo Objects (MACHOs; Alcock et al. 1995), the first phase of Optical Gravitational Lensing Experiment (OGLE-I; Udalski et al. 1992), and the first phase of Microlensing Observations in Astrophysics (MOA-I; Bond et al. 2001) surveys, it was difficult to detect planetary signals lasting of an order of 1 day or less by the survey experiments. To meet the cadence requirement for planet detections, Gould \& Loeb (1992) proposed an observational mode, in which widefield surveys with a low cadence monitor a large area of the sky mainly to detect lensing events, and follow-up experiments conduct high-cadence observations for a small number of lensing events detected by the surveys using a network of 
multiple narrow-field telescopes. However, this mode of observations had the drawback that only a handful of lensing events could be monitored by follow-up observations. Combined with the low probability of planetary perturbations, this implied a low planet detection rate for this phase of the experiments. In fact, for the first several years, there were no securely detected planets using this mode, although there was one tentative detection in the event MACHO 98-BLG-35 (Rhie et al. 2000).

The first three microlensing detections were found using the survey+follow-up strategy. In the first event, OGLE 2003BLG-235/MOA 2003-BLG-53 (Bond et al. 2004), the planet was found by the surveys, but the MOA survey carried out additional follow-up observations in response to the planetary anomaly. The next two planets, OGLE-2005-BLG-071Lb (Udalski et al. 2005) and OGLE-2005-BLG-390Lb (Beaulieu et al. 2006), were both found through extensive follow-up observations of known microlensing events that were initiated before the planetary anomaly began. The discovery of OGLE2005-BLG-071Lb provided a practical lesson in the value of high-magnification events (Griest \& Safizadeh 1998) for detecting planets through follow-up observations.

In the following years, the planet detection rate using the survey+follow-up mode was substantially increased by focusing on events with very high magnifications. Several factors contributed to the increase of the detection rate. First, the planet detection efficiency for high-magnification events is high. This is because a planet located in the lensing zone of its host always induces a small central caustic near the position of the host, and during a high-magnification event, the source passes close to the central caustic. This yields a high probability that a planet will produce a perturbation and also confines that perturbation to a short duration of time while the event is highly magnified, not throughout the whole event. As a result, the time of the planetary signal, i.e., the peak of the light curve, can be predicted in advance and enable one to efficiently use resources for follow-up observations. By contrast, predicting the time of a planetary signal through other channels is difficult. Finally, highly magnified source stars are bright enough to be observed with small-aperture telescopes, down to submeter amateur-class telescopes, and this enables one to maximize available telescopes for follow-up observations, e.g., OGLE-2005BLG-071 (Udalski et al. 2005; Dong et al. 2009). Thus, the planets detected from the survey+follow-up experiments were detected mainly through the high-magnification channel, and this led to the bias toward central-caustic perturbations.

The current planetary lensing experiments are in the second phase, in which lensing events are observed by high-cadence surveys. The observational cadence of the lensing surveys in this phase has greatly increased with the employment of largeformat cameras yielding very wide fields of view. The MOA experiment entered a new phase (MOA-II) by upgrading its instrument with a new wide-field camera composed of ten $2 \mathrm{k} \times 4 \mathrm{k}$ chips yielding a $2.2 \mathrm{deg}^{2}$ field of view (Sumi et al. 2013). The OGLE survey is in its fourth phase (OGLE-IV) using a $1.4 \mathrm{deg}^{2}$ camera composed of $322 \mathrm{k} \times 4 \mathrm{k}$ chips (Udalski et al. 2015). The Korea Microlensing Telescope Network (KMTNet) survey, which commenced its full operation in 2016 (Kim et al. 2016), utilizes three globally distributed telescopes, each of which has a camera with a $4.0 \mathrm{deg}^{2}$ field of view. Being able to cover a large area of sky from a single exposure, the observational cadence of the current survey experiments now reaches $\Gamma \sim 4 \mathrm{hr}^{-1}$ toward the dense bulge fields. This enables planet detections without additional follow-up observations.

With the operation of the high-cadence surveys, the detection rate of planets is rapidly increasing. One important reason for the rapid increase of the detection rate is that planets can be detected not only through the central-caustic channel but also through the additional planetary-caustic channel. Planets are detected through the planetary-caustic channel as anomalies produced by the source's approach close to the planetary caustic, which denotes one of the two sets of planet-induced caustics lying away from the host. The planetary caustic lies at a position with a separation from the host of $s-1 / s$, and thus planetary signals produced by this caustic can appear at any part of the lensing light curve depending on the planet-host separation $s$ (normalized to the angular Einstein radius $\theta_{\mathrm{E}}$ ). The planetary caustic is substantially larger than the central caustic, and thus the probability of a planetary perturbation is higher. Another importance of detecting planets through the planetarycaustic channel is that interpreting the planetary signal is usually not subject to the close-wide degeneracy (Griest \& Safizadeh 1998; Dominik 1999), which causes ambiguity in estimating the planet-host separations for most planets detected through the central-caustic channel.

In this paper, we present the analysis of two planetary microlensing events, OGLE-2018-BLG-0567 and OGLE2018-BLG-0962, for which planets are both detected through a planetary-caustic channel. For both events, the signatures of the planets were densely and continuously covered by two high-cadence lensing surveys, and this leads us to unambiguously interpret the planetary signals.

\section{Observation}

The two planetary events were observed by the two lensing surveys conducted by the OGLE and KMTNet groups. The OGLE survey uses the $1.3 \mathrm{~m}$ telescope that is located at the Las Campanas Observatory in Chile. The KMTNet survey utilizes three $1.6 \mathrm{~m}$ telescopes that are located at the Siding Spring Observatory in Australia (KMTA), the Cerro Tololo Interamerican Observatory in Chile (KMTC), and the South African Astronomical Observatory in South Africa (KMTS). The global distribution of the KMTNet telescopes makes it possible to continuously monitor the events. In both surveys, observations were mainly conducted in the $I$ band, and a fraction of the images were taken in the $V$ band to determine the color of the microlensed source stars.

OGLE-2018-BLG-0567， (R.A., decl. $)_{\mathrm{J} 2000}=(17: 56: 04.42$, $-27: 59: 13.6)$, or $(l, b)=\left(1^{\circ} .99,-1^{\circ} .49\right)$ in Galactic coordinates, was discovered on 2018 April 14 by the OGLE Early Warning System (EWS; Udalski 2003). The event was independently found by the KMTNet survey as KMT-2018BLG-0890 from its event-finding algorithm (Kim et al. 2018). The observational cadence for the event is $\Gamma=1 \mathrm{hr}^{-1}$ for OGLE and $\Gamma=2 \mathrm{hr}^{-1}$ for KMTNet.

OGLE-2018-BLG-0962 was discovered by the OGLE EWS on 2018 June 2. It is located at (R.A ., decl. $)_{\mathrm{J} 2000}=(17: 52: 41.95$, $-32: 18: 33.3)$ or Galactic coordinates of $(l, b)=(-2.11$, $\left.-3^{\circ} .04\right)$. The OGLE cadence for this direction is $3-10$ times per night. The KMTNet collaboration also observed the event, with designation KMT-2018-BLG-2071, located in their two overlapping fields (BLG41 and BLG22). In combination, the KMTNet observations have a frequency of $\Gamma=3 \mathrm{hr}^{-1}$ for KMTC and $\Gamma=2.25 \mathrm{hr}^{-1}$ for KMTS and KMTA. 

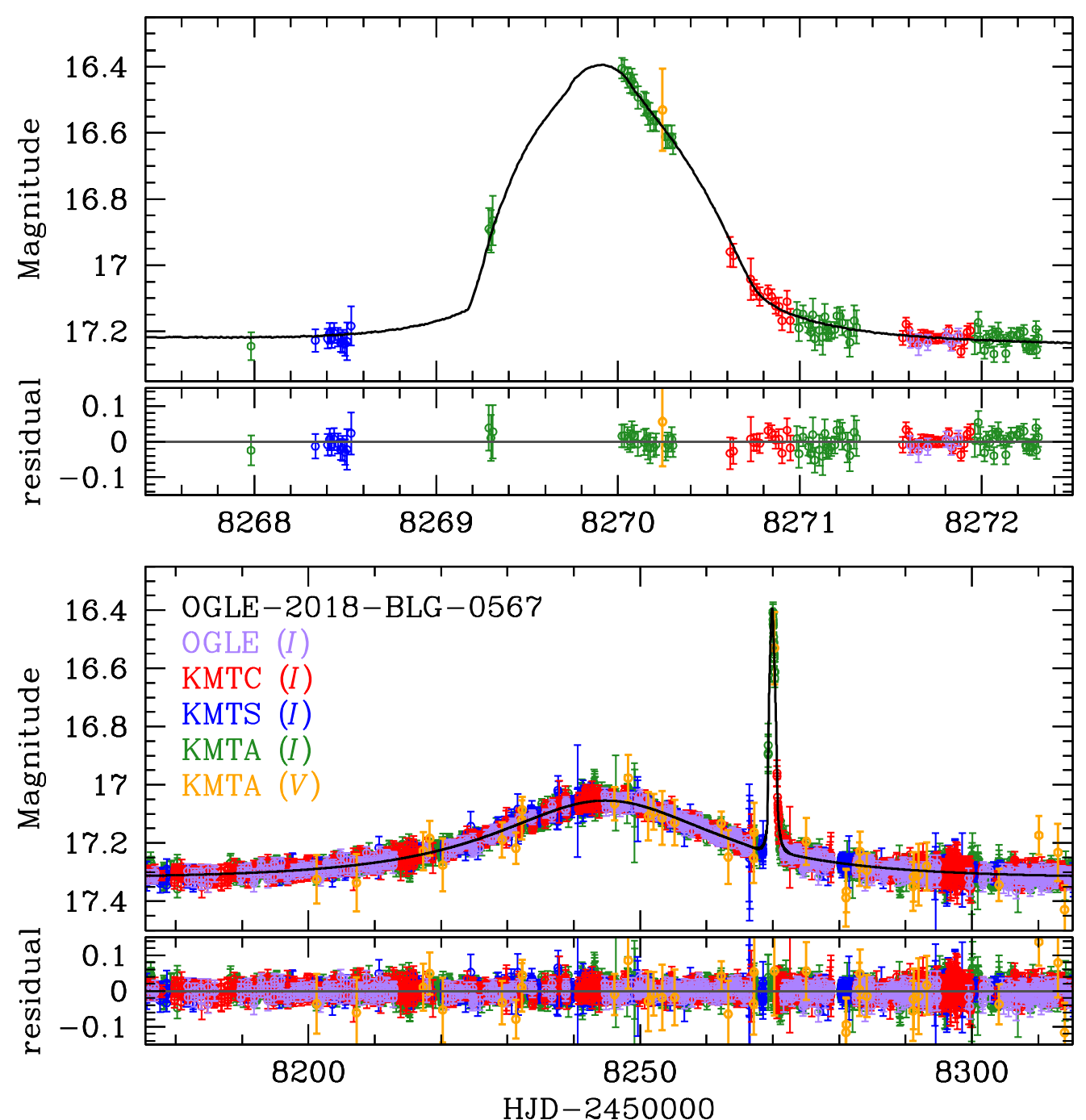

Figure 1. Light curve of OGLE-2018-BLG-0567. The black solid curve on the data is the best-fit 2L1S solution. The upper panel shows the enlarged view of the planet-induced anomaly centered on $\mathrm{HJD}^{\prime} \sim 8270$. The second and fourth panels show the residuals from the solution. The lensing parameters of the solution are listed in Table 1 and the caustic geometry is shown in Figure 3. Note that we use the $V$-band data only for the source color measurement.

(The data used to create this figure are available.)

For both events, the data sets were reduced based on the image-subtraction methodology (Tomaney \& Crotts 1996; Alard \& Lupton 1998), specifically Albrow et al. (2009) for KMTNet and Woźniak (2000) for OGLE. The photometric error bars were then readjusted following the prescription presented in Yee et al. (2012). We note that for the source color measurement, we additionally carried out pyDIA (Albrow 2017) reductions for a subset of the KMTNet data, which simultaneously returns the light curve and field-star photometry on the same system.

\section{Light Curve Analysis}

Figures 1 and 2 show the light curves of OGLE-2018-BLG0567 and OGLE-2018-BLG-0962, respectively. It is found that the two events share various characteristics in common. First, the apparent peak magnifications of the baseline single-lens single-source (1L1S) light curves are not high: $A_{\text {peak }} \sim 1.6$ for OGLE-2018-BLG-0567 and $A_{\text {peak }} \sim 4.9$ for OGLE-2018-BLG0962. Second, the light curves of both events exhibit strong short-term positive anomalies from the baseline 1L1S curves.
Third, the anomalies appear when the 1L1S-model lensing magnifications are low. All these characteristics strongly suggest that the anomalies are produced by source crossings over the planetary caustics induced by planetary companions to the lenses. We, therefore, start with a binary-lens single-source (2L1S) modeling of the events under the interpretation that the events were produced by lenses composed of two masses, $M_{1}$ and $M_{2}$.

The standard 2L1S modeling requires one to include seven fitting parameters to describe an observed light curve. The first three are the Paczyński (1986) parameters $\left(t_{0}, u_{0}, t_{\mathrm{E}}\right)$, which are respectively the time of closest source approach to the lens, the impact parameter (scaled to $\theta_{\mathrm{E}}$ ), and the event timescale. The next three $(s, q, \alpha)$ describe the binary-lens geometry: the projected binary separation (scaled to $\theta_{\mathrm{E}}$ ), the binary mass ratio $\left(q=M_{2} / M_{1}\right)$, and the angle between the source trajectory and the binary axis as measured in a clockwise sense, respectively. The last describes the source radius $\rho=\theta_{*} / \theta_{\mathrm{E}}$, where $\theta_{*}$ is the angular source radius. See Figure 6 of Jung et al. (2015) for a graphical presentation of the parameters. 

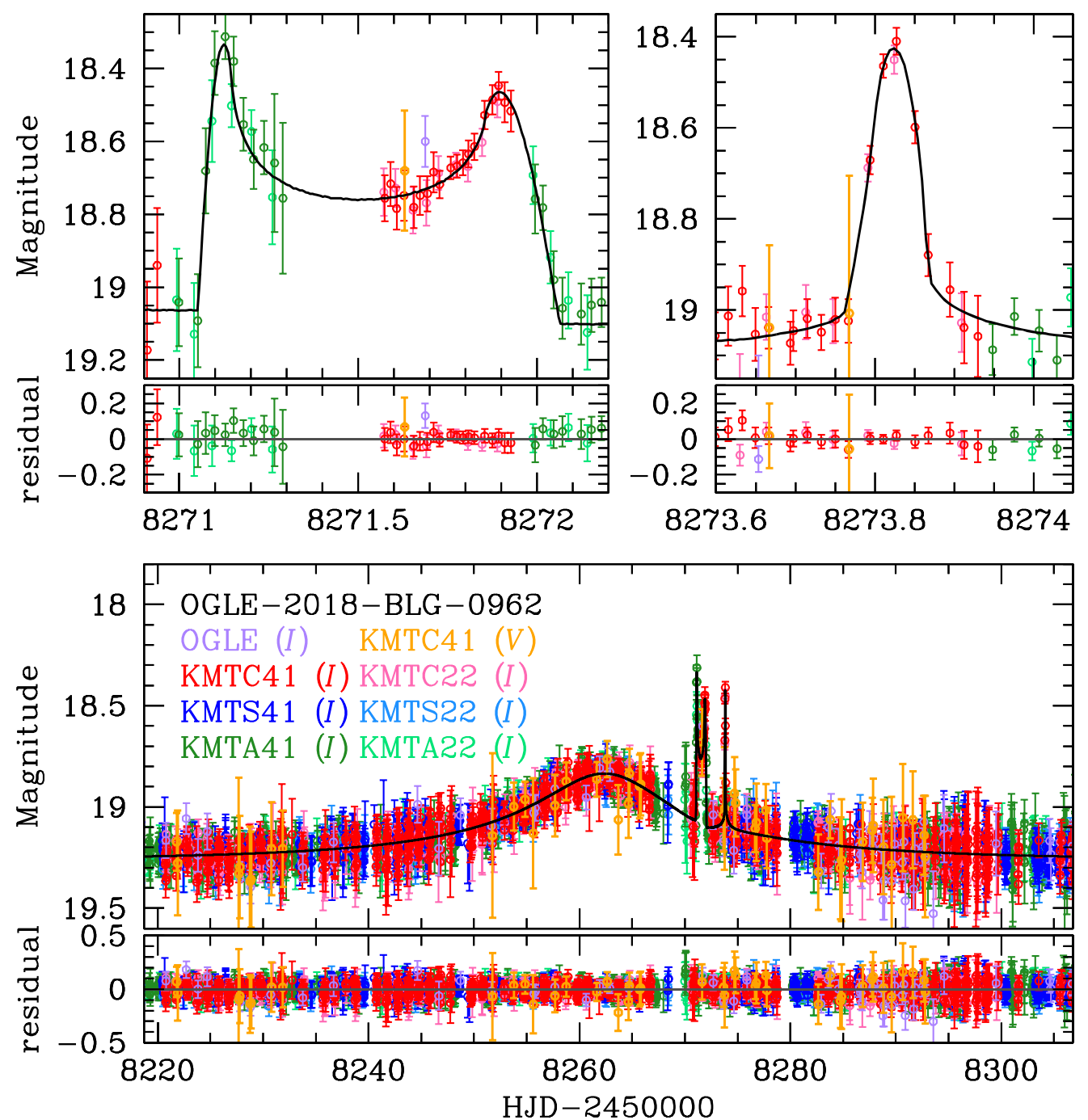

Figure 2. Light curve of OGLE-2018-BLG-0962. The upper panels show the close-up views of the regions around HJD' $\sim 8271.5$ (left) and HJD' $\sim 8273.8$ (right) when the planet-induced perturbations occur. The lensing parameters of the 2L1S solution are listed in Table 1 and the caustic geometry is shown in Figure 4.

(The data used to create this figure are available.)

The modeling is conducted following the procedure described in Jung et al. (2015). In the first step, we carry out grid searches for the binary parameters $(s, q, \alpha)$, with $100 \times 100 \times 21$ different grid points. The ranges of the grid parameters are $-1 \leqslant \log s \leqslant 1,-5 \leqslant \log q \leqslant 0$, and $0 \leqslant$ $\alpha \leqslant 2 \pi$. At each grid, we fix $(s, q)$ and find the remaining parameters using Markov Chain Monte Carlo (MCMC) $\chi^{2}$ minimization. In this modeling, the initial values of the parameters $\left(t_{0}, u_{0}, t_{\mathrm{E}}\right)$ are given as the values estimated from a 1L1S fit for the data excluding the anomaly. The initial value of the normalized source radius is estimated from the causticcrossing timescale, which is related to the event timescale by $t_{*}=\rho t_{\mathrm{E}}$. Here we use inverse ray shooting (Kayser et al. 1986; Schneider \& Weiss 1987) to compute the finite-source lensing magnifications. The flux values from the source, $f_{\mathrm{S}, i}$, and blend, $f_{\mathrm{B}, i}$, for the data set obtained from the $i$ th observatory are estimated by $f_{i}(t)=f_{\mathrm{S}, i} A(t)+f_{\mathrm{B}, i}$, where $f_{i}$ is the observed flux. Once local solutions are found from the first-round modeling, we refine the individual locals by releasing all fitting parameters and allowing them to be free parameters in an MCMC.
From the modeling, it is found that the observed lensing light curves of both events are well described by unique 2L1S models, in which the mass ratios between $M_{1}$ and $M_{2}$ are in the planetary regime. In addition, the planetary perturbations in both light curves are not subject to the close-wide degeneracy. The estimated binary parameters are $(s, q)=(1.81$, $\left.1.24 \times 10^{-3}\right)$ for OGLE-2018-BLG-0567 and $(s, q)=(1.25$, $2.38 \times 10^{-3}$ ) for OGLE-2018-BLG-0962. The full lensing parameters and their uncertainties are presented in Table 1. The model curves of the solutions are drawn over the data points in Figures 1 and 2 for OGLE-2018-BLG-0567 and OGLE-2018BLG-BLG-0962, respectively.

In Figures 3 and 4, we present the lens-system configurations of the individual events, showing the source trajectory with respect to the lens components and resulting caustics. From the configurations, it is found that the anomalies of both events are produced by the source crossing over the planetary caustic of the lens system. For OGLE-2018-BLG-0567, the source size is comparable to the caustic size, and thus the detailed causticcrossing features, two caustic spikes and a U-shape trough region between the spikes, were smeared out by finite-source 

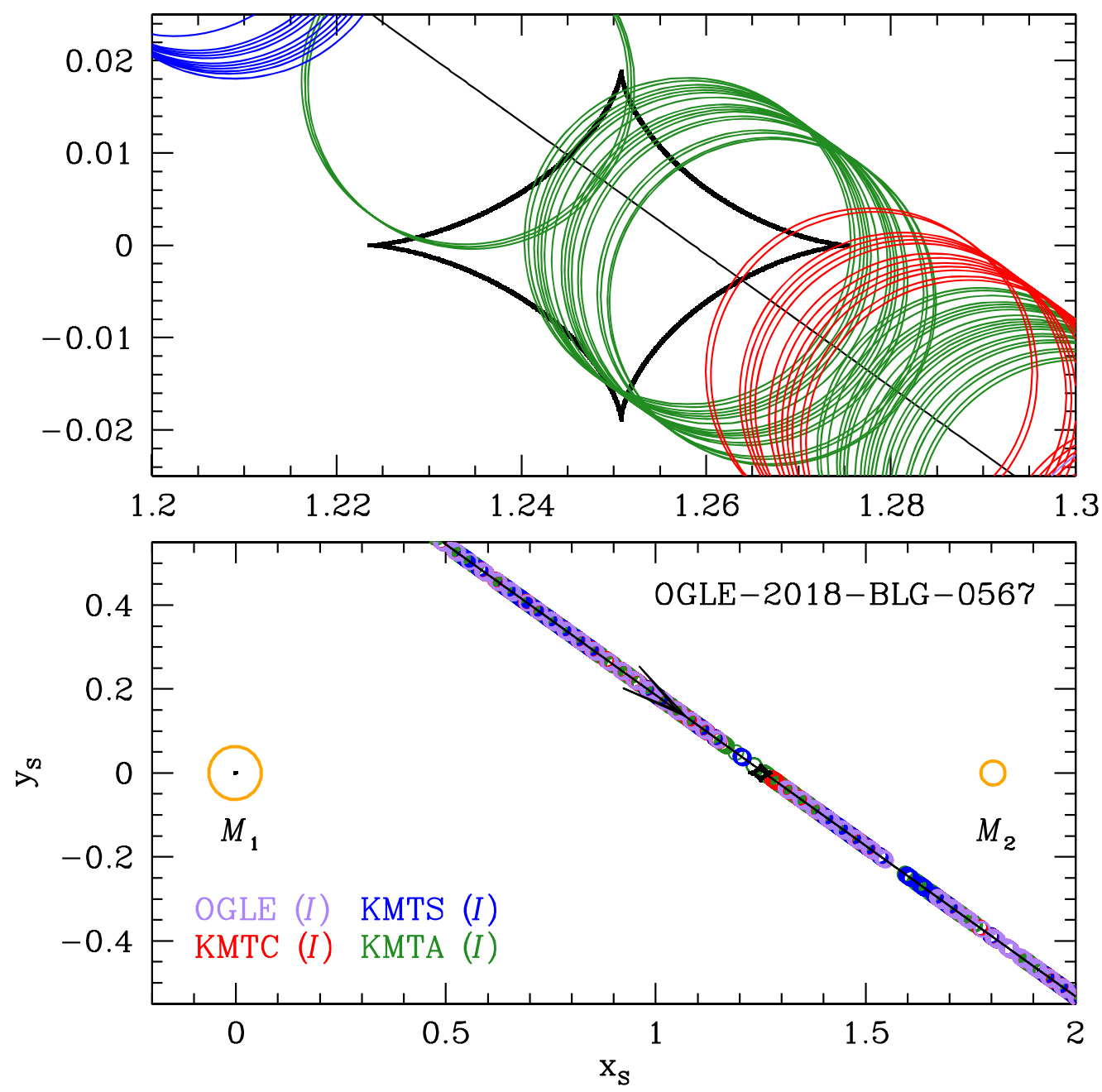

Figure 3. Caustic geometry of OGLE-2018-BLG-0567. The line with an arrow is the source trajectory relative to the binary-lens axis. The open circles (scaled by the normalized source radius $\rho$ ) on the trajectory are the source positions at the times of observations. The two orange circles are the positions of binary-lens masses $\left(M_{1}\right.$ and $M_{2}$ ). In each panel, the cuspy closed curve drawn in black represents the caustic. The upper panel shows the enlarged view of the planetary caustic. Lengths are scaled to the angular Einstein radius of the lens system.

Table 1

Lensing Parameters

\begin{tabular}{lcc}
\hline \hline Parameters & OGLE-2018-BLG-0567 & OGLE-2018-BLG-0962 \\
\hline$\chi_{\text {tot }}^{2} /$ dof & $8677.3 / 9252$ & $6892.1 / 6833$ \\
$t_{0}\left(\mathrm{HJD}^{\prime}\right)$ & $8244.845 \pm 0.025$ & $8262.494 \pm 0.053$ \\
$u_{0}$ & $0.733 \pm 0.026$ & $0.207 \pm 0.032$ \\
$t_{\mathrm{E}}(\mathrm{days})$ & $24.641 \pm 1.064$ & $28.739 \pm 0.298$ \\
$s$ & $1.806 \pm 0.019$ & $1.246 \pm 0.027$ \\
$q\left(10^{-3}\right)$ & $1.240 \pm 0.068$ & $2.375 \pm 0.076$ \\
$\alpha(\mathrm{rad})$ & $0.623 \pm 0.045$ & $0.590 \pm 0.044$ \\
$\rho\left(10^{-3}\right)$ & $17.675 \pm 0.831$ & $1.137 \pm 0.048$ \\
$f_{\mathrm{S}}$ & $0.842 \pm 0.035$ & $0.039 \pm 0.007$ \\
$f_{\mathrm{B}}$ & $1.026 \pm 0.035$ & $0.274 \pm 0.007$ \\
\hline
\end{tabular}

effects. For OGLE-2018-BLG-0962, on the other hand, the caustic is much bigger than the source size, and thus the detailed caustic-crossing feature of the anomaly is well delineated. It is found that the first part of the anomaly, centered at $\mathrm{HJD}^{\prime}(=\mathrm{HJD}-2,450,000$ days $) \sim 8271.5$, was produced by the source passing over the two caustic segments that flank the inner cusp (on the binary axis) of the planetary caustic, and the second part, centered at $\mathrm{HJD}^{\prime} \sim 8273.8$, was generated by the source passage over the adjacent (off-axis) cusp.
We investigate the possibility of other interpretations of the events. Especially for OGLE-2018-BLG-0567, the short-term perturbation might, in principle, be produced by a second source (1L2S) with a large flux ratio (Gaudi 1998). Hence, we conduct an additional modeling of the event with the 1L2S interpretation (Jung et al. 2017). We search for the solution with eight fitting parameters: $2 \times\left(t_{0}, u_{0}, \rho\right)$ for the two sources, I-band flux ratio $q_{F, I}$, and a shared timescale $t_{\mathrm{E}}$. The results are listed in Table 2. We find that the 1L2S solution is disfavored by $\Delta \chi^{2}>500$. In addition, the 1L2S model not only provides a worse fit to the peak of the perturbation, but also fails to recover the decrease in magnitude seen before and after the peak. See Figure 5.

We check the feasibility of constraining the microlens parallax, $\pi_{\mathrm{E}}$, by conducting an additional modeling of the light curve. In this modeling, we simultaneously consider the microlens-parallax and lens-orbital effects (Gould 1992; Dominik 1998), because the light curve deviations induced by the parallax effect can be correlated with the deviations induced by the lens-orbital motion (Batista et al. 2011). For OGLE-2018-BLG-0567, we find that it is difficult to accurately determine $\pi_{\mathrm{E}}$ not only because the improvement of the fit, $\Delta \chi^{2} \sim 9$, is very minor, but also because the parallax vector 

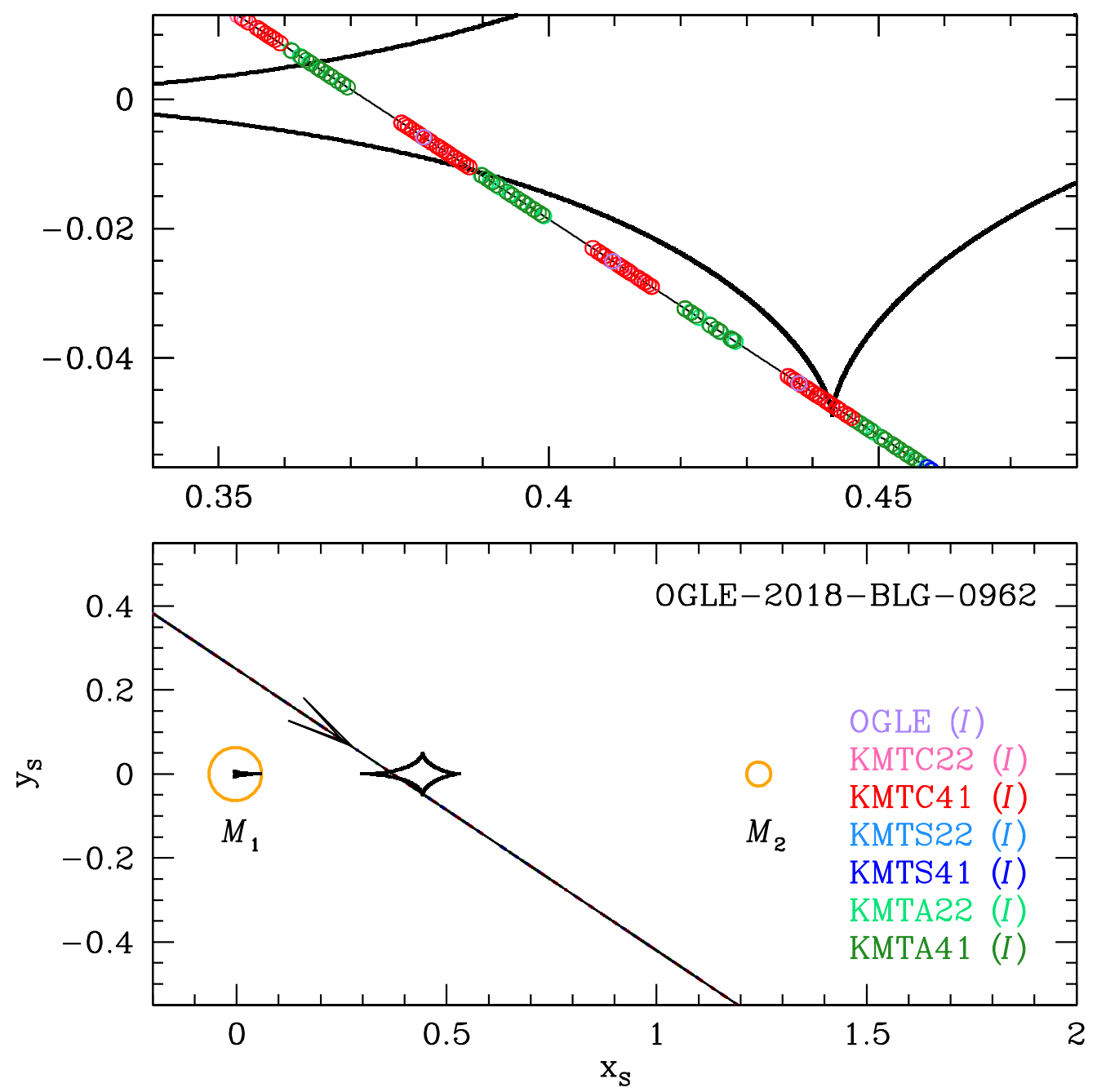

Figure 4. Caustic geometry of OGLE-2018-BLG-0962. Notations are identical to those of Figure 3.

Table 2

Binary Source Model for OGLE-2018-BLG-0567

\begin{tabular}{lc}
\hline \hline Parameters & $1 \mathrm{~L} 2 \mathrm{~S}$ \\
\hline$\chi^{2} /$ dof & $9223.0 / 9251$ \\
$t_{0,1}\left(\mathrm{HJD}^{\prime}\right)$ & $8244.323 \pm 0.043$ \\
$u_{0,1}$ & $0.705 \pm 0.039$ \\
$t_{0,2}\left(\mathrm{HJD}^{\prime}\right)$ & $8670.134 \pm 0.010$ \\
$u_{0,2}\left(10^{-5}\right)$ & $2.603 \pm 35.093$ \\
$t_{\mathrm{E}}($ days $)$ & $24.088 \pm 0.868$ \\
$\rho_{1}$ & $\cdots$ \\
$\rho_{2}\left(10^{-3}\right)$ & $6.295 \pm 0.344$ \\
$q_{F, I}\left(10^{-3}\right)$ & $7.520 \pm 0.461$ \\
$f_{\mathrm{s}}$ & $0.795 \pm 0.082$ \\
$f_{\mathrm{b}}$ & $1.073 \pm 0.082$ \\
\hline
\end{tabular}

does not converge to a specific value. For OGLE-2018-BLG0962, the fit improvement, $\Delta \chi^{2} \sim 19$, is somewhat bigger than that of OGLE-2018-BLG-0567, but the modeling yields an abnormally large parallax value, $\left(\pi_{\mathrm{E}, N}, \pi_{\mathrm{E}, E}\right)=(-3.44 \pm 0.57$, $-1.05 \pm 0.42)$, suggesting the possibility that the higher-order parameters are correlated with the noise in the data rather than the parallax signal being real (e.g., Jung et al. 2019). These imply that it is difficult to constrain the parallax parameters for both events.

\section{Physical Parameters}

The results in Table 1 show that for both events, the normalized source radii are precisely measured. This enables us to estimate $\theta_{\mathrm{E}}=\theta_{*} / \rho$, provided that $\theta_{*}$ is measured. Then, we can use the estimated $\theta_{\mathrm{E}}$ to constrain the lens total mass $M$ and distance $D_{\mathrm{L}}$ as given by

$$
\theta_{\mathrm{E}}^{2} \equiv \kappa M \pi_{\mathrm{rel}} ; \quad \pi_{\mathrm{rel}}=\mathrm{au}\left(\frac{1}{D_{\mathrm{L}}}-\frac{1}{D_{\mathrm{S}}}\right),
$$

where $\kappa \equiv 4 G /\left(c^{2}\right.$ au $) \simeq 8.14$ mas $/ M_{\odot}, \pi_{\text {rel }}$ is the lens-source relative parallax, and $D_{\mathrm{S}}$ is the source distance. Hence, we evaluate $\theta_{*}$ using the method of Yoo et al. (2004).

We first estimate the intrinsic source color $(V-I)_{0, S}$ and magnitude $I_{0, \mathrm{~S}}$. We do this in the following ways. First, we investigate the KMTNet images and identify the data containing the $V$ - and $I$-band images taken around the peak of the event. We next reduce the KMTA (for OGLE-2018-BLG0567) and KMTC41 (for OGLE-2018-BLG-0962) data using pyDIA software, and calibrate the pyDIA reductions to the standard Johnson-Cousins system using the OGLE-III catalog (Szymański et al. 2011). We then construct a $(V-I, I)$ colormagnitude diagram (CMD) with field stars around the source, and find the position of the red clump centroid (RCC), i.e., $(V-I, I)_{\mathrm{RCC}}$. Based on the best-fit model, we next conduct a 


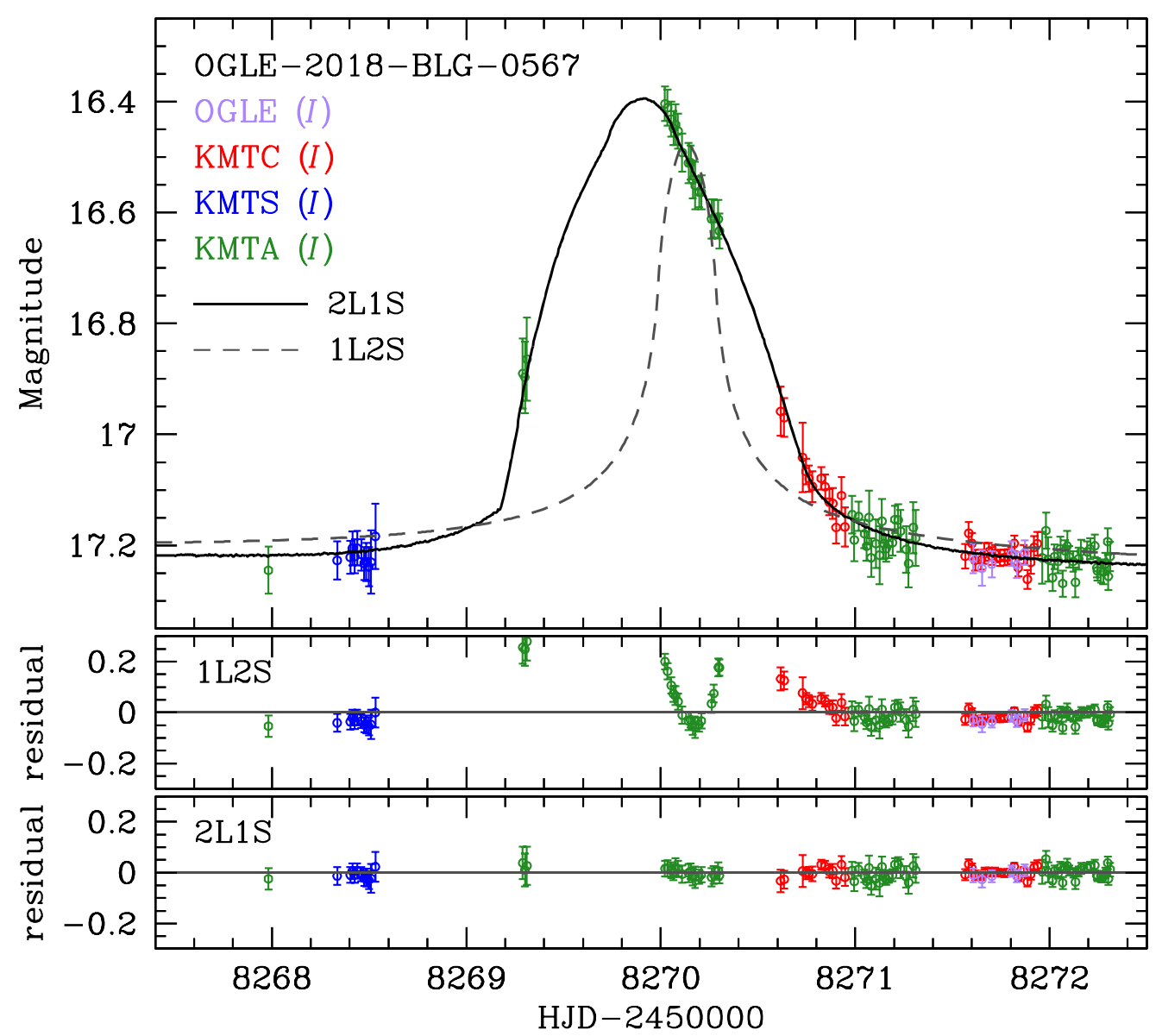

Figure 5. Light curve of the 1L2S model for OGLE-2018-BLG-0567. The dashed gray and solid black lines are the best-fit models from the 1L2S and 2L1S interpretations, respectively. The lower two panels show the residuals from the two models.

2L1S modeling including the pyDIA $V$ - and $I$-band data sets, and estimate their flux values to find the source location $(V-I, I)_{\mathrm{S}}$ in the CMD. Figure 6 shows the source and RCC positions in the CMDs for the individual events. We then measure the offset $\Delta(V-I, I)=(V-I, I)_{\mathrm{S}}-(V-I, I)_{\mathrm{RCC}}$. Finally, we find the intrinsic source position as

$$
(V-I, I)_{0, \mathrm{~S}}=\Delta(V-I, I)+(V-I, I)_{0, \mathrm{RCC}},
$$

where $(V-I, I)_{0, \mathrm{RCC}}$ is the intrinsic RCC position measured from independent observations (Bensby et al. 2013; Nataf et al. 2013). Here we assume that the source star experiences the same amount of extinction as the RCC. In Table 3, we list our estimated values of $(V-I, I)_{\mathrm{S}},(V-I, I)_{\mathrm{RCC}},(V-I, I)_{0, \mathrm{RCC}}$, and $(V-I, I)_{0, \mathrm{~S}}$.

We now derive $\theta_{*}$ based on the estimated intrinsic source position. For this, we apply $(V-I)_{0, S}$ to the $V I K$ relation (Bessell \& Brett 1988) to find $(V-K)_{0, \mathrm{~S}}$. We then obtain $\theta_{*}$ from the $(V-K)_{0, \mathrm{~S}}-\theta_{*}$ relations, specifically Kervella et al. (2004a) for OGLE-2018-BLG-0567 and Kervella et al. (2004b) for OGLE-2018-BLG-0962. We note that we add a 5\% error to $\theta_{*}$ to consider the uncertainty of the intrinsic RCC position and the color/surface brightness conversion. From the measured $t_{\mathrm{E}}$ and $\rho$, we then find $\theta_{\mathrm{E}}$ and the lens-source relative proper motion, $\mu_{\text {rel }}=\theta_{\mathrm{E}} / t_{\mathrm{E}}$. The estimated values of $\theta_{*}, \theta_{\mathrm{E}}$, and $\mu_{\text {rel }}$ are also listed in Table 3.

For both events, we are unable to constrain the microlensparallax vector $\boldsymbol{\pi}_{\mathrm{E}}$. This implies that we cannot directly derive
$M$ and $D_{\mathrm{L}}$ by

$$
M=\frac{\theta_{\mathrm{E}}}{\kappa \pi_{\mathrm{E}}} ; \quad D_{\mathrm{L}}=\frac{\mathrm{au}}{\pi_{\mathrm{E}} \theta_{\mathrm{E}}+\pi_{\mathrm{S}}}
$$

from the microlensing data (Gould 2000). Here $\pi_{\mathrm{S}}=\mathrm{au} / D_{\mathrm{S}}$. Hence, we estimate the lens properties based on a Bayesian analysis with Galactic model priors.

The Galactic model is constructed based on a mass function (MF), a density profile (DP), and a velocity distribution (VD). For the MF, we use the models presented in Jung et al. (2018). For the DP, we use the Han \& Gould (2003) model for the bulge and the Robin et al. (2003) model for the disk, respectively. We note that the former is normalized based on the star count results of Holtzman et al. (1998), while the latter is normalized by the local column density $\Sigma\left(D_{\mathrm{L}}=0\right)=$ $\Sigma_{\text {disk }, 0}=36 M_{\odot} \mathrm{pc}^{-2}$ from Han \& Gould (2003).

The bulge VD is modeled based on stars in the Gaia catalog (Gaia Collaboration et al. 2016, 2018). That is, we first find red giant stars in the catalog within $2^{\prime}$ centered on the event location. We then derive their mean velocity and its dispersion in Galactocentric Cartesian coordinates $(x, y, z)$ as defined in Han \& Gould (1995). ${ }^{17}$

For the disk VD, we adopt Gaussian forms of $f\left(v_{y}\right.$, $\left.v_{z}\right)=f\left(v_{y}\right) f\left(v_{z}\right)$ from Han \& Gould (1995), which we then modify to consider the change in the matter distribution. We do

\footnotetext{
${ }^{17}$ We note that we use 541 (for OGLE-2018-BLG-0567) and 587 (for OGLE2018-BLG-0962) red giant stars to estimate the bulge velocity distribution.
} 


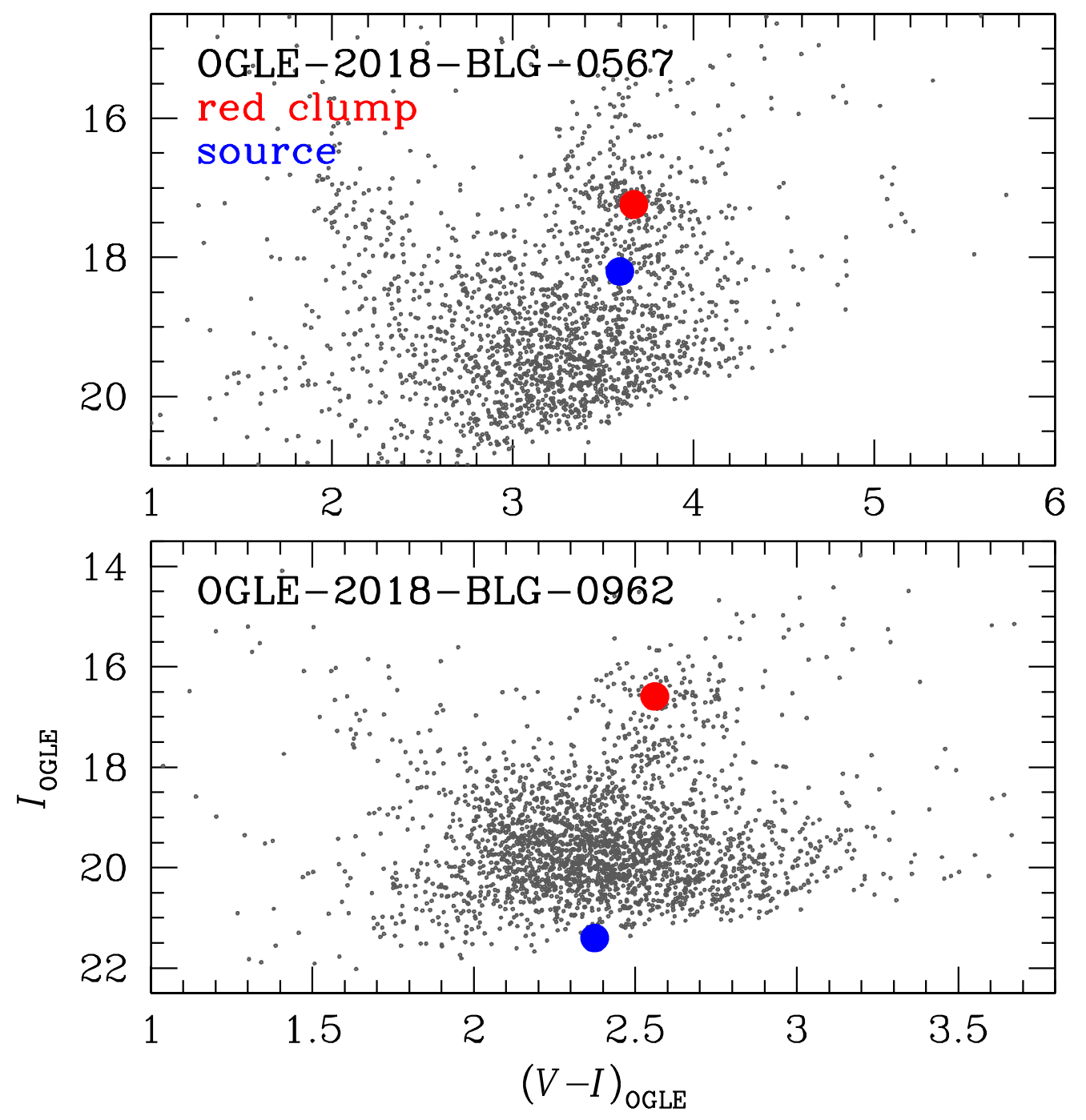

Figure 6. Color-magnitude diagrams of OGLE-2018-BLG-0567 (upper panel) and OGLE-2018-BLG-0962 (lower panel). In each panel, the CMD is constructed using stars in the $2^{\prime} \times 2^{\prime}$ field centered on the event location based on KMTNet pyDIA photometry calibrated to the OGLE-III catalog (Szymański et al. 2011). The blue and red circles are the positions of the source and red clump centroid, respectively.

Table 3

Source Star and Lens Properties

\begin{tabular}{lcc}
\hline \hline Parameters & OGLE-2018-BLG-0567 & OGLE-2018-BLG-0962 \\
\hline$(V-I, I)_{\mathrm{S}}$ & $(3.59 \pm 0.10,18.20 \pm 0.01)$ & $(2.37 \pm 0.08,21.40 \pm 0.03)$ \\
$(V-I, I)_{\mathrm{RCC}}$ & $(3.67 \pm 0.08,17.24 \pm 0.08)$ & $(2.56 \pm 0.12,16.59 \pm 0.11)$ \\
$(V-I, I)_{0, \mathrm{RCC}}$ & $(1.06,14.37)$ & $(1.06,14.56)$ \\
$(V-I, I)_{0, \mathrm{~S}}$ & $(0.98 \pm 0.13,15.33 \pm 0.08)$ & $(0.87 \pm 0.14,19.37 \pm 0.11)$ \\
$\theta_{*}(\mu \mathrm{as})$ & $3.769 \pm 0.507$ & $0.504 \pm 0.076$ \\
$\theta_{\mathrm{E}}(\mathrm{mas})$ & $0.213 \pm 0.030$ & $0.443 \pm 0.069$ \\
$\mu_{\text {rel }}\left(\right.$ mas $\left._{\mathrm{yr}}{ }^{-1}\right)$ & $3.161 \pm 0.451$ & $5.639 \pm 0.879$ \\
\hline
\end{tabular}

this in two ways. First, we introduce an asymmetric drift $v_{\text {ad }}$ to the $y$-direction velocity $v_{y}$. That is, $v_{\mathrm{ad}}\left(D_{\mathrm{L}}\right)=$ $\left(0.5 \sigma_{x y z, 0}^{2} / \bar{v}_{y}\right)\left[\Sigma\left(D_{\mathrm{L}}\right) / \Sigma_{\text {disk }, 0}\right]^{1 / 2}$, where $\bar{v}_{y}=220 \mathrm{~km} \mathrm{~s}^{-1}$ is the mean $y$-direction velocity, $\sigma_{x y z, 0}^{2}=\sigma_{x, 0}^{2}+\sigma_{y, 0}^{2}+\sigma_{z, 0}^{2}$, and $\left(\sigma_{x, 0}, \sigma_{y, 0}, \sigma_{z, 0}\right)=(34,30,20) \mathrm{km} \mathrm{s}^{-1}$ are the mean velocity dispersions along the $(x, y, z)$ directions (in the solar neighborhood), respectively. The $y$-direction velocity at a
Table 4

Physical Parameters

\begin{tabular}{lcc}
\hline \hline Parameters & OGLE-2018-BLG-0567 & OGLE-2018-BLG-0962 \\
\hline$M_{1}\left(M_{\odot}\right)$ & $0.25_{-0.13}^{+0.27}$ & $0.54_{-0.28}^{+0.33}$ \\
$M_{2}\left(M_{\mathrm{J}}\right)$ & $0.32_{-0.17}^{+0.34}$ & $1.34_{-0.70}^{+0.82}$ \\
$a_{\perp}(\mathrm{au})$ & $2.72_{-0.59}^{+0.53}$ & $3.59_{-1.12}^{+0.81}$ \\
$D_{\mathrm{L}}(\mathrm{kpc})$ & $7.06_{-1.15}^{+0.93}$ & $6.50_{-1.75}^{+1.06}$ \\
\hline
\end{tabular}

given line-of-sight distance $\left(D_{\mathrm{L}}\right)$ is then calculated by $v_{y}\left(D_{\mathrm{L}}\right)=\bar{v}_{y}-v_{\mathrm{ad}}\left(D_{\mathrm{L}}\right)$. Second, we subsequently modify the velocity dispersion as $\sigma_{y}\left(D_{\mathrm{L}}\right)=\sigma_{y, 0}\left[\Sigma\left(D_{\mathrm{L}}\right) / \Sigma_{\text {disk, }, 0}\right]^{1 / 2}$ for the $y$-direction and $\sigma_{z}\left(D_{\mathrm{L}}\right)=\sigma_{z, 0}\left[\Sigma\left(D_{\mathrm{L}}\right) / \Sigma_{\text {disk, } 0}\right]^{1 / 2}$ for the $z$-direction.

We now carry out the Bayesian analysis with the constraints $\left(t_{\mathrm{E}}, \theta_{\mathrm{E}}\right)$. For this, we follow the procedure of Jung et al. (2018). The estimated lens properties for the individual events are listed in Table 4. The corresponding posterior probabilities for $M_{1}$ and $D_{\mathrm{L}}$ are shown in Figure 7. We find that the host mass is 

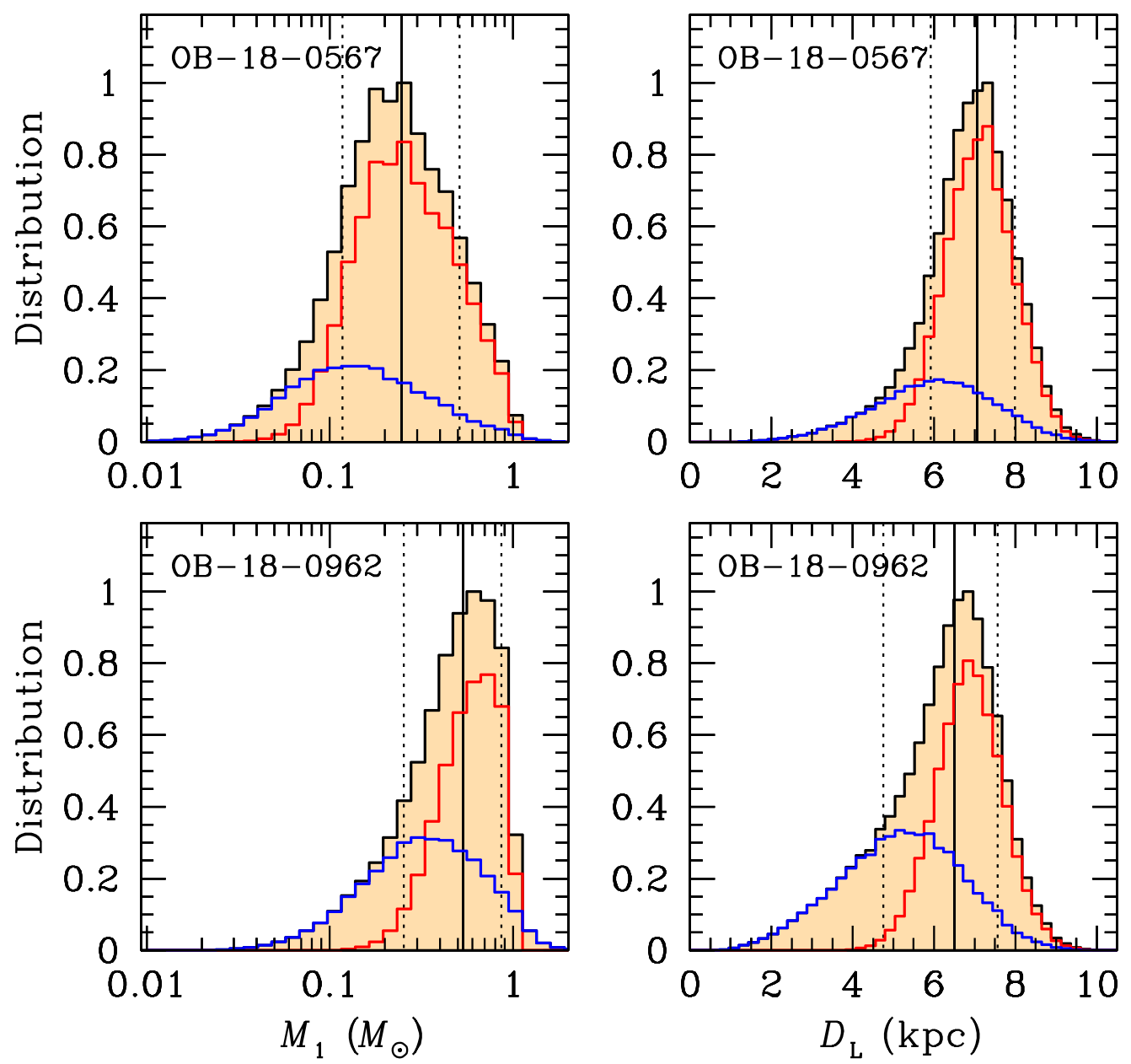

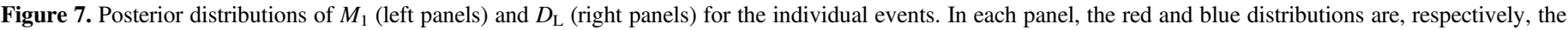

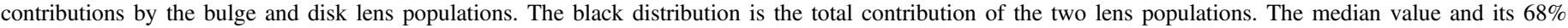
confidence interval are represented by the vertical solid and two dotted lines, respectively.

$M_{1}=0.25_{-0.13}^{+0.27} M_{\odot}$ for OGLE-2018-BLG-0567 and $M_{1}=$ $0.54_{-0.28}^{+0.33} M_{\odot}$ for OGLE-2018-BLG-0962. The planet masses $\left(M_{2}=q M_{1}\right)$ and the projected planet-host separations $\left(a_{\perp}=\right.$ $\left.s D_{\mathrm{L}} \theta_{\mathrm{E}}\right)$ of the individual events are then estimated to $\left(M_{2}, a_{\perp}\right)=\left(0.32_{-0.17}^{+0.34} M_{\mathrm{J}}, 2.72_{-0.59}^{+0.53} \mathrm{au}\right)$ and $\left(M_{2}, a_{\perp}\right)=$ $\left(1.34_{-0.70}^{+0.82} M_{\mathrm{J}}, 3.59_{-1.12}^{+0.81} \mathrm{au}\right)$, respectively. These estimates suggest that the two planetary systems are likely composed of M-type dwarfs and giant planets lying beyond the snow line (Kennedy \& Kenyon 2008). Here, the snow line is the location in the protoplanetary disk where icy material can condense and where giant planets are thought to be formed (Ida \& Lin 2004). The distance to the planet is $D_{\mathrm{L}}=7.06_{-1.15}^{+0.93} \mathrm{kpc}$ for OGLE2018-BLG-0567 and $D_{\mathrm{L}}=6.50_{-1.75}^{+1.06} \mathrm{kpcfor}$ OGLE-2018BLG-0962, indicating that they are likely to be in or near the Galactic bulge.

\section{Microlensing Planets in the $(\log s, \log q)$ Plane}

Our two survey-only microlensing planets are detected from the perturbations caused by the planetary caustics (see Figures 3 and 4). In particular, the planetary perturbation of OGLE-2018-BLG-0567 was generated by a "Hollywood" geometry (Gould 1997), in which the source size contributes strongly to, or dominates, the anomaly cross-section relative to the size of the caustic. These detections prove the capability of the high-cadence surveys for detecting planets through the planetary-caustic channel.

Figure 8 illustrates the positions of OGLE-2018-BLG0567Lb and OGLE-2018-BLG-0962Lb in the $(\log s, \log q)$ plane along with other microlensing planets. ${ }^{18}$ This figure shows that OGLE-2018-BLG-0567Lb is located in a previously underpopulated region. The two green solid lines indicate the boundary between resonant and nonresonant caustics (Schneider \& Weiss 1986; Dominik 1999). For planets just outside of the resonance region, the excess magnification pattern (from the underlying 1L1S magnification) extends out all the way between the central and planetary caustics. This implies that the effective cross-section of these caustics for a planet-induced perturbation and their lensing behavior are similar to those of resonant caustics. Recently, Yee et al. (2021) classified such kinds of caustics as the "near-resonant," specifically a set of caustics that has a excess magnification contour that connects the central and planetary caustics and has at least $10 \%$ excess magnification along the entire caustic ridges. The two green dashed lines are the boundary of nearresonant caustics. Yee et al. (2021) empirically estimated that at a fixed $q$, the maximum size of a $10 \%$ deviation corresponds to

$\overline{18 \text { https://exoplanetarchive.ipac.caltech.edu }}$ 


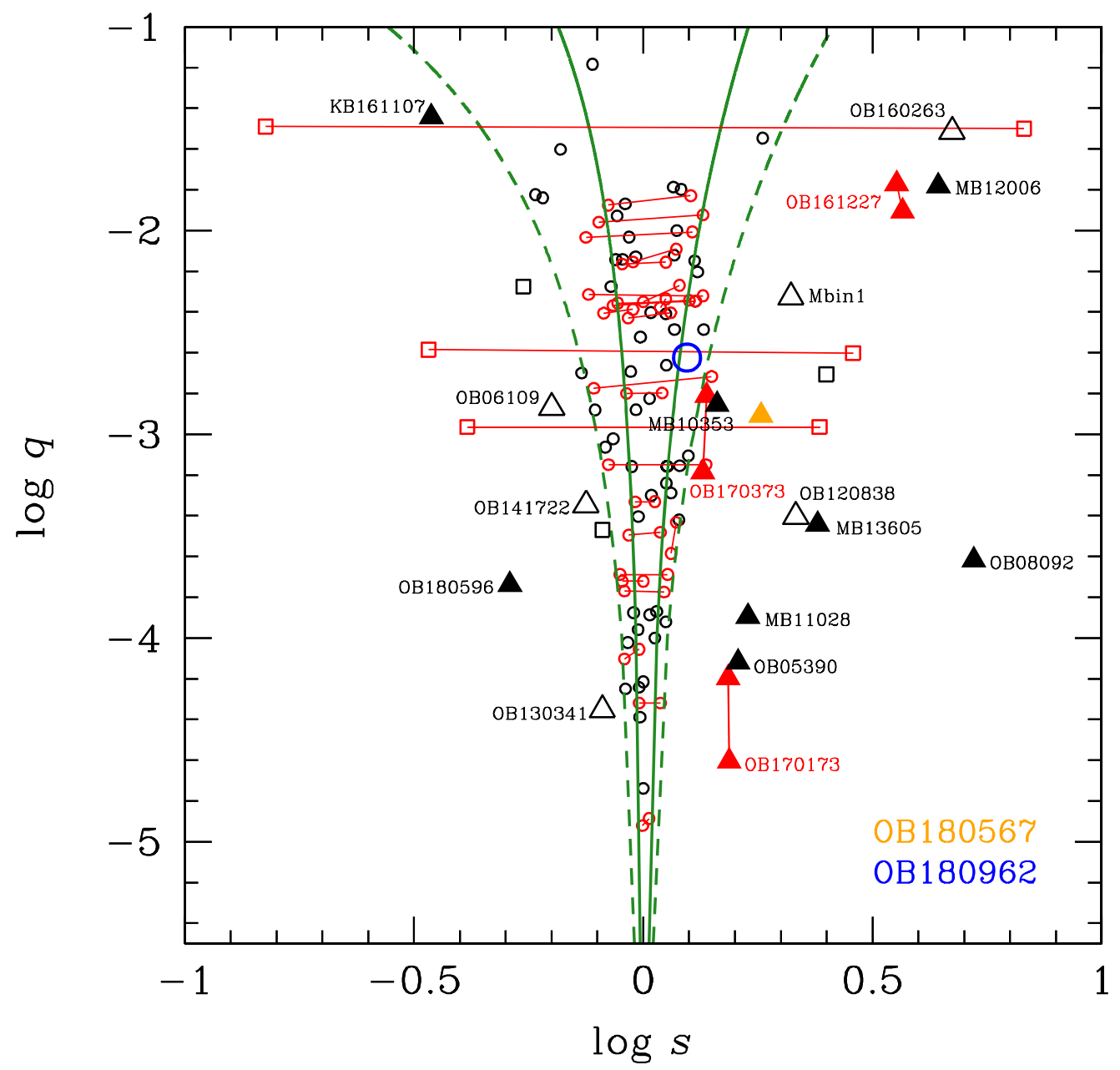

Figure 8. Microlensing planets in the $(\log s, \log q)$ plane, adapted from Figure 9 of Yee et al. (2021). Planets (except for our two planets) are colored by the number of solutions: black for one solution and red (with connected line) for degenerate solutions. The two planets OGLE-BLG-2018-BLG-0567Lb and OGLE-2018-BLG0962 Lb are coded by yellow and blue, respectively. Their shapes indicate the caustic structure giving rise to the planetary perturbation: circles for resonant/nearresonant, squares for central, and triangles for planetary caustics. The filled triangles are the planets from the Hollywood events. The two green solid and dashed lines are the boundary of resonant and near-resonant caustics, respectively. We note that for compactness, we compress the planet names, e.g., OGLE-2018-BLG-0567Lb to OB180567.

$\sim 3 \log s_{\mathrm{r}, \mathrm{c}}$ for $s<1$ (close) and $\sim 1.8 \log s_{\mathrm{r}, \mathrm{w}}$ for $s>1$ (wide), where $s_{\mathrm{r}, \mathrm{c}}$ and $s_{\mathrm{r}, \mathrm{w}}$ are the boundary values of $s$ between resonant and nonresonant caustics (Dominik 1999). Planets (except for our two planets) with a single and multiple solutions are coded by black and red (with connected lines) colors, respectively. OGLE-2018-BLG-0567Lb and OGLE-2018BLG-0962Lb are marked by yellow and blue colors, respectively. The shape of the symbols represent the type of caustics that yield the planetary perturbation: circles for resonant/near-resonant, squares for central, and triangles for planetary caustics. The filled triangles are the planets found in the Hollywood events.

Figure 8 shows that the majority of planets are located inside the near-resonant boundary rather than being due to planetary caustics. The bias toward resonant caustics mainly comes from the relatively large size of resonant caustics (scaled as $q^{1 / 3}$ ) compared to that of planetary caustics (scaled as $q^{1 / 2}$; Dominik 1999; Han 2006; Yee et al. 2021). There is also a bias against planetary caustics due to the observational strategy of earlier microlensing experiments that was focused on high-magnification events (Griest \& Safizadeh 1998; Gould et al. 2010).
Only 24 planets are placed outside the near-resonant boundary and 18 planets among them are detected from the perturbations produced by clearly isolated planetary caustics. ${ }^{19}$ We find that most of these planetary-caustic planets (12 planets) are found in the Hollywood events and they are located in high-cadence observational fields of the lensing surveys. This proves the capability of the Hollywood strategy of following big stars to find planets (Gould 1997). The majority of the Hollywood planets are located in the region $s>1$. This is mainly due to the difference in the size of planetary caustics. For $s>1$, there is one four-sided planetary caustic. For $s<1$,

\footnotetext{
19 The corresponding planetary-caustic events are OGLE-2005-BLG-390 (Beaulieu et al. 2006), MOA-bin-1 (Bennett et al. 2012), OGLE-2006-BLG109 (Gaudi et al. 2008; Bennett et al. 2010), OGLE-2008-BLG-092 (Poleski et al. 2014), MOA-2010-BLG-353 (Rattenbury et al. 2015), MOA-2011-BLG028 (Skowron et al. 2016), MOA-2012-BLG-006 (Poleski et al. 2017), OGLE2012-BLG-0838 (Poleski et al. 2020), OGLE-2013-BLG-0341 (Gould et al. 2014), MOA-2013-BLG-605 (Sumi et al. 2016), OGLE-2014-BLG-1722 (Suzuki et al. 2018), OGLE-2016-BLG-0263 (Han et al. 2017), OGLE-2016BLG-1227 (Han et al. 2020), KMT-2016-BLG-1107 (Hwang et al. 2019), OGLE-2017-BLG-0173 (Hwang et al. 2018), OGLE-2017-BLG-0373 (Skowron et al. 2018), OGLE-2018-BLG-0596 (Jung et al. 2019), and OGLE-2018BLG-0962 (this work).
} 
on the other hand, there are two triangular planetary caustics and each of which size is much smaller than that of $s>1$. In addition, the planetary signals from the these smaller planetary caustics tend to be more significantly diminished by the finitesource effects (Gould \& Gaucherel 1997). As a result, the wideplanetary caustic has a larger effective cross-section and therefore higher sensitivity for finding planets.

\section{Summary and Conclusions}

We present the discovery of two cold, giant planets orbiting M-dwarfs in two events, OGLE-2018-BLG-0567 and OGLE2018-BLG-0962. Both events clearly showed deviations from the 1L1S model, caused by the presence of a companion to the lens host with precisely measured planet/host mass ratios of $(1.24 \pm 0.07) \times 10^{-3}$ and $(2.38 \pm 0.08) \times 10^{-3}$, respectively. In both events, the finite-source effects are clearly detected, but the microlens-parallax effects are not meaningfully constrained. Hence, we constrain the lens properties using the Bayesian analysis. From this, we estimate planet masses of $0.32_{-0.17}^{+0.34} M_{\mathrm{J}}$ for OGLE-2018-BLG-0567Lb and $1.34_{-0.70}^{+0.82} M_{\mathrm{J}}$ for OGLE2018-BLG-0962Lb, and their physical projected separations of $2.72_{-0.59}^{+0.53}$ au and $3.59_{-1.12}^{+0.81}$ au, respectively. These planets likely belong to a class of giant planets orbiting M-dwarfs outside the snow line. The detection rate of microlensing planets has rapidly increased with the advent of high-cadence lensing surveys, and thus, planets presented here and future detections will expand our understanding of the planet population around M-dwarfs.

The planet hosts can be precisely constrained by future high-resolution imaging with adaptive optics (AO) mounted on $30 \mathrm{~m}$ class telescopes. That is, they have only a small probability of being nonluminous (see Figure 7). The Bayesian estimates suggest that the dereddened $H$-band magnitude of the host is $H_{0}=21.79_{-1.85}^{+1.79}$ for OGLE-2018-BLG-0567 and $H_{0}=19.63_{-1.67}^{+1.89}$ for OGLE-2018-BLG-0962 (Pecaut $\&$ Mamajek 2013). In addition, both events have $\mu_{\text {rel }}>$

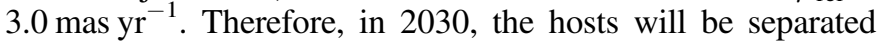
from the microlensed source by $\Delta \theta \gtrsim 40$ mas.

This research has made use of the KMTNet system operated by the Korea Astronomy and Space Science Institute (KASI) and the data were obtained at three host sites of CTIO in Chile, SAAO in South Africa, and SSO in Australia. Work by C.H. was supported by the grants of National Research Foundation of Korea (2017R1A4A1015178 and 2019R1A2C2085965). The OGLE has received funding from the National Science Centre, Poland, grant MAESTRO 2014/14/A/ST9/00121 to A.U.

\section{ORCID iDs}

Youn Kil Jung (1) https://orcid.org/0000-0002-0314-6000 Cheongho Han (iD https://orcid.org/0000-0002-2641-9964 Andrzej Udalski (i) https://orcid.org/0000-0001-5207-5619 Jennifer C. Yee (iD https://orcid.org/0000-0001-9481-7123 Michael D. Albrow (iD https://orcid.org/0000-0003-3316-4012 Sun-Ju Chung (iD https://orcid.org/0000-0001-6285-4528 Kyu-Ha Hwang (iD https://orcid.org/0000-0002-9241-4117 Yoon-Hyun Ryu (i) https://orcid.org/0000-0001-9823-2907 In-Gu Shin (iD https://orcid.org/0000-0002-4355-9838 Yossi Shvartzvald (ib https://orcid.org/0000-0003-1525-5041 Wei Zhu (iD https://orcid.org/0000-0003-4027-4711
Weicheng Zang (iD https://orcid.org/0000-0001-6000-3463 Hyoun-Woo Kim (iD https://orcid.org/0000-0001-8263-1006 Seung-Lee Kim (1) https://orcid.org/0000-0003-0562-5643 Chung-Uk Lee (iD https://orcid.org/0000-0003-0043-3925 Byeong-Gon Park (i) https://orcid.org/0000-0002-6982-7722 Richard W. Pogge (iD https://orcid.org/0000-0003-1435-3053 Przemek Mróz (1D https://orcid.org/0000-0001-7016-1692 Michał K. Szymański (i) https://orcid.org/0000-00020548-8995

Jan Skowron (iD https://orcid.org/0000-0002-2335-1730 Igor Soszyński (ib https://orcid.org/0000-0002-7777-0842 Paweł Pietrukowicz (iD https://orcid.org/0000-0002-2339-5899 Szymon Kozłowski ii https://orcid.org/0000-00034084-880X

Krzystof Ulaczyk (D) https://orcid.org/0000-0001-6364-408X Patryk Iwanek (i) https://orcid.org/0000-0002-6212-7221

\section{References}

Alard, C., \& Lupton, R. H. 1998, ApJ, 503, 325

Albrow, M. D. 2017, MichaelDAlbrow/pyDIA: Initial release on github, V1.0.0, Zenodo, doi:10.5281/zenodo.268049

Albrow, M. D., Horne, K., Bramich, D. M., et al. 2009, MNRAS, 397, 2099 Alcock, C., Allsman, R. A., Axelrod, T. S., et al. 1995, ApJ, 445, 133

Batista, V., Gould, A., Dieters, S., et al. 2011, A\&A, 529, 102

Beaulieu, J.-P., Bennett, D. P., Fouqué, P., et al. 2006, Natur, 439, 437 Bennett, D. P., Rhie, S. H., Nikolaev, S., et al. 2010, ApJ, 713, 837 Bennett, D. P., Sumi, T., Bond, I. A., et al. 2012, ApJ, 757, 119 Bensby, T., Yee, J. C., Feltzing, S., et al. 2013, A\&A, 549, 147 Bessell, M. S., \& Brett, J. M. 1988, PASP, 100, 1134

Bond, I. A., Abe, F., \& Dodd, R. J. 2001, MNRAS, 327, 868

Bond, I. A., Udalski, A., Jaroszyński, M., et al. 2004, ApJ, 606, L155

Dominik, M. 1998, A\&A, 329, 361

Dominik, M. 1999, A\&A, 349, 108

Dong, S., Gould, A., Udalski, A., et al. 2009, ApJ, 695, 970

Gaia Collaboration, Brown, A. G. A., Vallenari, A., et al. 2018, A\&A, 616, A1

Gaia Collaboration, Prusti, T., de Bruijne, J. H. J., et al. 2016, A\&A, 595, A1

Gaudi, B. S. 1998, ApJ, 506, 533

Gaudi, B. S. 2012, ARA\&A, 50, 411

Gaudi, B. S., Bennett, D. P., Udalski, A., et al. 2008, Sci, 319, 927

Gould, A. 1992, ApJ, 392, 442

Gould, A. 1997, in Variables Stars and the Astrophysical Returns of the Microlensing Surveys, ed. R. Ferlet, J.-P. Maillard, \& B. Raban (Gif-surYvette: Editions Frontieres), 125

Gould, A. 2000, ApJ, 542, 785

Gould, A., Dong, S., Gaudi, B. S., et al. 2010, ApJ, 720, 1073

Gould, A., \& Gaucherel, C. 1997, ApJ, 477, 580

Gould, A., \& Loeb, A. 1992, ApJ, 396, 104

Gould, A., Udalski, A., Shin, I.-G., et al. 2014, Sci, 345, 46

Griest, K., \& Safizadeh, N. 1998, ApJ, 500, 37

Han, C. 2006, ApJ, 638, 1080

Han, C., \& Gould, A. 1995, ApJ, 447, 53

Han, C., \& Gould, A. 2003, ApJ, 592, 172

Han, C., Udalski, A., Gould, A., et al. 2017, AJ, 154, 133

Han, C., Udalski, A., Gould, A., et al. 2020, AJ, 159, 91

Holtzman, J. A., Watson, A. M., Baum, W. A., et al. 1998, AJ, 115, 1946 Hwang, K.-H., Ryu, Y.-H., Kim, H.-W., et al. 2019, AJ, 157, 23

Hwang, K.-H., Udalski, A., Shvartzvald, Y., et al. 2018, AJ, 155, 20 Ida, S., \& Lin, D. N. C. 2004, ApJ, 616, 567

Jung, Y. K., Gould, A., Udalski, A., et al. 2019, AJ, 158, 28

Jung, Y. K., Udalski, A., Gould, A., et al. 2018, AJ, 155, 219

Jung, Y. K., Udalski, A., Sumi, T., et al. 2015, ApJ, 798, 123 Jung, Y. K., Udalski, A., Yee, J. C., et al. 2017, AJ, 153, 129 Kayser, R., Refsdal, S., \& Stabell, R. 1986, A\&A, 166, 36

Kennedy, G. M., \& Kenyon, S. J. 2008, ApJ, 673, 502

Kervella, P., Bersier, D., Mourard, D., et al. 2004a, A\&A, 428, 587

Kervella, P., Thévenin, F., Di Folco, E., \& Ségransan, D. 2004b, A\&A, 426, 297

Kim, D.-J., Kim, H.-W., Hwang, K.-H., et al. 2018, AJ, 155, 76

Kim, S.-L., Lee, C.-U., Park, B.-G., et al. 2016, JKAS, 49, 37

Nataf, D. M., Gould, A., Fouqué, P., et al. 2013, ApJ, 769, 88

Paczyński, B. 1986, ApJ, 304, 1 
Pecaut, M. J., \& Mamajek, E. E. 2013, ApJS, 208, 9

Poleski, R., Skowron, J., Udalski, A., et al. 2014, ApJ, 795, 42

Poleski, R., Suzuki, D., Udalski, A., et al. 2020, AJ, 159, 261

Poleski, R., Udalski, A., Bond, I. A., et al. 2017, A\&A, 604A, 103

Rattenbury, N. J., Bennett, D. P., Sumi, T., et al. 2015, MNRAS, 454, 946

Rhie, S. H., Bennett, D. P., Becker, A. C., et al. 2000, ApJ, 533, 378

Robin, A. C., Reylé, C., Derriére, S., \& Picaud, S. 2003, A\&A, 409, 523

Schneider, P., \& Weiss, A. 1986, A\&A, 164, 237

Schneider, P., \& Weiss, A. 1987, A\&A, 171, 49

Skowron, J., Ryu, Y.-H., Hwang, K.-H., et al. 2018, AcA, 68, 43

Skowron, J., Udalski, A., Poleski, R., et al. 2016, ApJ, 820, 4

Sumi, T., Bennett, D. P., Bond, I. A., et al. 2013, ApJ, 778, 150

Sumi, T., Udalski, A., Bennett, D. P., et al. 2016, ApJ, 825, 112
Suzuki, D., Bennett, D. P., Udalski, A., et al. 2018, AJ, 155, 263

Szymański, M. K., Udalski, A., Soszyński, I., et al. 2011, AcA, 61, 83

Tomaney, A. B., \& Crotts, A. P. S. 1996, AJ, 112, 2872

Udalski, A. 2003, AcA, 53, 291

Udalski, A., Jaroszyński, M., Paczyński, B., et al. 2005, ApJL, 628, L109

Udalski, A., Szymański, M., Kałużny, J., Kubiak, M., \& Mateo, M. 1992, AcA, 42,253

Udalski, A., Szymański, M. K., \& Szymański, G. 2015, AcA, 65, 1 Woźniak, P. R. 2000, AcA, 50, 421

Yee, J. C., Shvartzvald, Y., Gal-Yam, A., et al. 2012, ApJ, 755, 102

Yee, J. C., Zang, W., Udalski, A., et al. 2021, arXiv:2101.04696

Yoo, J., DePoy, D. L., Gal-Yam, A., et al. 2004, ApJ, 603, 139 